\title{
Descripción del uso de medicamentos gastroprotectores en pacientes con polifarmacia en una población colombiana, a partir de bases de datos transaccionales
}

\author{
Descriptive Analysis of Transactional Database Date on the Use of \\ Gastroprotective Drugs in Patients With Polypharmacy in a Colombian Population
}

Álvaro Vallejos N., MD, Msc, ${ }^{1}$ Laura Maldonado C., ${ }^{2}$ Juan Camilo Calvache V., MD, ${ }^{3}$ William Hernandez D., ${ }^{4}$ Sandra Torres R., ${ }^{5}$ Dieric Diaz S., MD Esp. ${ }^{6}$

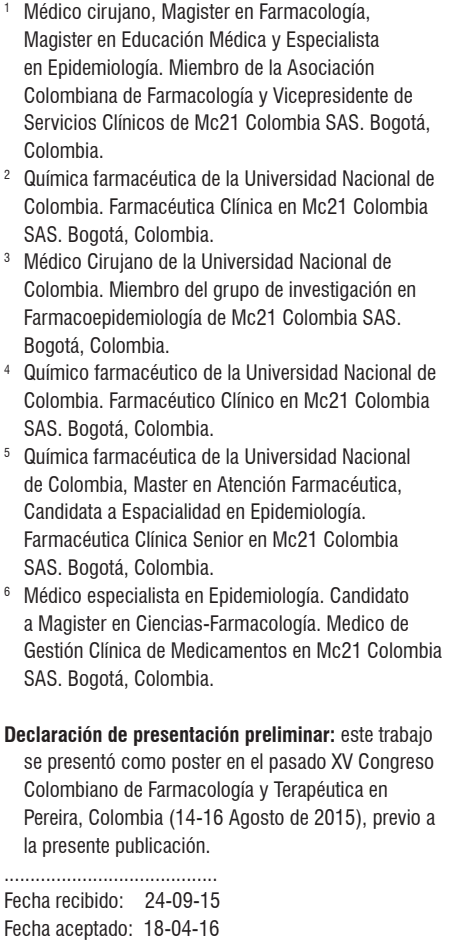

Declaración de presentación preliminar: este trabajo se presentó como poster en el pasado XV Congreso Colombiano de Farmacología y Terapéutica en Pereira, Colombia (14-16 Agosto de 2015), previo a la presente publicación.

Fecha recibido: $24-09-15$ Fecha aceptado: 18-04-16

\section{Resumen}

Objetivo: el objetivo de este estudio fue describir y analizar los patrones de prescripción de fármacos antiulcerosos, los factores asociados a su prescripción y su costo en pacientes ambulatorios polimedicados en un período de 6 meses, en una Entidad Promotora de Salud (EPS) colombiana a nivel nacional. Metodología: estudio descriptivo retrospectivo de corte transversal, basado en registros electrónicos de prescripción de medicamentos ambulatorios de 2458447 afiliados. Se incluyeron pacientes con prescripción de 5 o más fármacos por mes y se excluyeron aquellos cuyos registros transaccionales no tenían información completa para su análisis. Se evaluó la asociación entre prescripción de fármacos antiulcerosos y factores que justifican su prescripción mediante los odds ratio (OR) calculados a partir de un modelo de regresión logística. Resultados: de 2458447 afiliados, 60671 pacientes estuvieron polimedicados mensualmente; $40 \%$ tuvieron fármacos antiulcerosos y $70 \%$ fármacos gastrolesivos. De los gastroprotegidos, $47 \%$ fueron adultos mayores y $12 \%$ tuvieron asociado diagnóstico de riesgo gastrointestinal superior. Gastroprotección no justificada en $35 \%$ de los pacientes polimedicados, representando $\$ 75$ millones de pesos colombianos (COP) mensuales. No hubo asociación estadística entre la prescripción de antiulcerosos y factores que justifiquen su prescripción (OR: 1,13; IC 95\%: 1,00-1,27). Conclusión: ante la falta de asociación entre la prescripción de fármacos antiulcerosos y los factores que la justifican, es probable que su prescripción se haya realizado por la polifarmacia per se. Se recomienda optimizar la gastroprotección y reservarla a pacientes con más de un gastrolesivo y riesgo gastrointestinal superior, estén estos polimedicados o no.

\section{Palabras clave}

Polifarmacia, antiulcerosos, inhibidores de la bomba de protones, antiinflamatorios no esteroideos, anciano.

\section{Abstract}

Objective: The objective of this study was to describe and analyze patterns of use of prescription ulcer drugs, factors associated with prescriptions by physicians, and costs for outpatients With polypharmacy at a Colombian healthcare promotion entity (EPS) over a six-month period. Methodology: This is a retrospective and descriptive cross-sectional study based on electronic records of drugs prescribed to 2,458,447 outpatient members. Patients who took five or more prescription drugs per month were included. Patient were excluded if their records in the transactional data were not complete enough for analysis. Associations between anti-ulcer drugs and factors justifying their prescription were evaluated by the odds ratio (OR) which was calculated from a logistic regression model. Results: Of the 2,458,447 affiliates of the EPS, on average 60,671 patients had polypharmacy each month: $40 \%$ used anti-ulcer drugs and $70 \%$ used gastro-damaging drugs. Of the gastroprotected patients, $47 \%$ were elderly and $12 \%$ had associated diagnoses of upper gastrointestinal risk. Gastroprotection was not justified in $35 \%$ of patients with polypharmacy. This represents 75 million Colombian pesos every month. There was no statistical association between prescription of anti-ulcer drugs and factors that justify their prescription (OR: $1.13 ; 95 \% \mathrm{Cl}: 1.00$ to 1.27). Conclusion: Given the lack of association between prescription of anti-ulcer drugs and factors which justify such prescriptions, it is likely that prescriptions contribute to polypharmacy per se. We recommended optimizing gastroprotection by reserving it for patients with more than one gastrointestinal lesion and with upper gastrointestinal risks whether or not they have polypharmacy.

\section{Keywords}

Polypharmacy, antiulcer, proton-pump inhibitor, NSAIDs, elderly. 


\section{INTRODUCCIÓN}

Más del 50\% de los pacientes mayores de 65 años presentan 2 o más enfermedades crónicas (1); la cronicidad y multimorbilidad conducen a la polifarmacia, definida como el uso de 5 o más medicamentos simultáneamente (2), aumentando la probabilidad de consulta por reacciones adversas a medicamentos (RAM) en un $88 \%$, incrementando costos para los pacientes y sistemas de salud $(3,4)$. Los medicamentos frecuentemente asociados a la polifarmacia son aquellos que actúan a nivel gastrointestinal, psicotrópico y cardiovascular (5); identificando dentro de los grupos farmacológicos más prescritos en pacientes crónicos: antihipertensivos (82,6\%), antiulcerosos $(73,8 \%)$, antitrombóticos $(70,3 \%)$ y antinflamatorios no esteroideos (AINE) (36,5\%) (6). En Corea, durante el año 2002, el $81,6 \%$ de los médicos internistas incluía antiulcerosos en sus prescripciones $(7,8)$, la inclusión de estos son un predictor de polifarmacia (9).

En el año 2014, se aclaró que únicamente requieren gastroprotección los pacientes en tratamiento con más de un fármaco gastrolesivo y factores de riesgo como historia previa de morbilidad gastrointestinal superior, diabetes mellitus o hipertensión arterial, edad mayor o igual a 65 años, terapia concomitante con otros fármacos gastrolesivos, comorbilidad grave y/o tratamiento crónico a dosis máximas de fármacos gastrolesivos $(10,11)$; lo cual indica que no todos los pacientes con fármacos gastrolesivos o con polifarmacia necesitan gastroprotección (12). Por ende, el objetivo de este estudio fue describir y analizar los patrones de prescripción de fármacos antiulcerosos, los factores asociados a su prescripción y su costo en pacientes ambulatorios polimedicados en un período de 6 meses, en una Entidad Promotora de Salud (EPS) colombiana a nivel nacional.

\section{MATERIAL Y MÉTODO}

Estudio observacional descriptivo retrospectivo de corte transversal sobre patrones de prescripción de fármacos antiulcerosos en pacientes polimedicados en una EPS con 2458447 afiliados al mes, en 420 Instituciones Prestadoras de Salud (IPS), de 136 municipios de Colombia, durante un período de 6 meses (septiembre 2014-febrero 2015), a partir de los registros electrónicos de prescripciones ambulatorias de la EPS. Se incluyeron los pacientes con prescripción de 5 o más fármacos al mes y se excluyeron aquellos cuyos registros transaccionales no tenían información completa para el análisis. Los registros electrónicos de prescripciones se obtuvieron a partir del Pharmacy Benefit Management (PBM) MC21 Colombia y a partir de esta información se analizaron las variables demográficas (edad), clínicas (diagnóstico médico) y farmacológicas (medicamentos prescritos por mes, medicamentos antiulcerosos y gastrolesivos prescritos por mes y costo de estos) de los pacientes.

La edad de los pacientes se agrupó en 4 categorías (paciente pediátrico (0-12 años), paciente adulto joven (13-44 años), paciente adulto maduro (45-64 años) y paciente anciano $(\geq$ 65 años); de acuerdo a lo establecido por el Grupo Nacional de Estadísticas en Salud de Medicare y Medicaid (13). El diagnóstico médico se agrupó en 5 categorías de acuerdo a los objetivos del estudio (patología gastrointestinal, que incluyó gastritis, duodenitis, esofagitis, úlceras gástrica, duodenal, péptica y gastroyeyunal, enfermedad por reflujo gastroesofágico (ERGE), varices esofágicas, gástricas y duodenales y cánceres del tracto gastrointestinal superior; patologías graves que incluyó septicemias, otros cánceres, hepatitis y otras hepatopatías, insuficiencia renal crónica y otras disfunciones renales, traumatismos graves y quemaduras graves; hipertensión arterial, diabetes mellitus y otros, para ello se utilizó la Clasificación Internacional de Enfermedades de la Organización Mundial de la Salud (OMS) en su décima versión (CIE-10).

Los medicamentos antiulcerosos y gastrolesivos fueron agrupados por grupos farmacológicos de acuerdo al Sistema de Clasificación Anatómica, Terapéutica y Química (ATC) de OMS; los grupos farmacológicos seleccionados como antiulcerosos fueron: antiácidos (A02A), antagonistas $\mathrm{H} 2$ (A02BA), prostaglandinas (A02BB), inhibidores de la bomba de protones (IBP) (A02BC) y los protectores directos de la mucosa (A02BX); y los grupos farmacológicos seleccionados como gastrolesivos fueron: antinflamatorios no esteroideos (AINE) (M01A), antiagregantes plaquetarios (B01AC), anticoagulantes (B01AA, B01AB, B01AE, B01AF, B01AX), inhibidores selectivos de la recaptación de serotonina (ISRS) $(\mathrm{N} 06 \mathrm{AB})$ y corticosteroides sistémicos (H02AB).

Se realizó un análisis descriptivo univariado y multivariado; se calcularon los coeficientes de correlación (Pearson y Phi) para medir la correlación existente entre la prescripción de antiulcerosos y los factores que justifican su prescripción; y los odds ratio (OR) mediante un modelo de regresión logística. La especificidad y sensibilidad del modelo se verificó mediante la curva ROC (área bajo la curva: 0,62 , el modelo predijo el comportamiento real en un $62 \%)$. El análisis se realizó en el software estadístico R (14), la manipulación de variables tipo fecha se realizó con el paquete "lubridate" (15), los procesos de transformación, extracción y agregación mediante el paquete "sqldf" (16) y las pruebas de bondad de ajuste del modelo mediante el paquete "pROC" (17). 


\section{RESULTADOS}

De 2458447 afiliados al mes, el 13\% (321 184) recibió medicamentos y de estos el 18\% (60 671) tenían polifarmacia; el 11\% (6 644) contaba con prescripciones de 10 o más fármacos y 49 pacientes llegaron a tener de 20 a 30 fármacos prescritos en un mismo mes. El 31\% de los pacientes polimedicados (18 808) fueron ancianos, 35\% (21 235) adultos maduros, 24\% (14 561) adultos jóvenes y $10 \%$ (6 067) pacientes pediátricos. La mayoría de pacientes estaban entre los 50 y los 70 años (Figura 1). A nivel nacional, el índice de polifarmacia (IP) (pacientes con polifarmacia/pacientes que recibieron medicamentos $x 100)$ se incrementó, se pasó de un 16\% en noviembre 2014 al 22\% en febrero de 2015; en este mes, 22 de cada 100 pacientes que recibieron medicamentos tenían prescritos 5 o más fármacos y 3 de cada 100 pacientes tenían prescritos 10 o más principios activos en el mismo mes.

El 40\% de los pacientes polimedicados (23 738) recibieron antiulcerosos. Los IBP fueron los más utilizados (19 157), seguidos por los antiácidos (Figura 2a). Del total de pacientes gastroprotegidos, el 17\% (4 060) tuvo prescrito antiulcerosos de 2 o más grupos farmacológicos simultáneamente; la asociación más frecuente fue IBP con antiácidos en el 65\% de los pacientes (1 644). Se observó la prescripción simultánea de IBP, antiácidos y sucralfato en 156 pacientes $(6 \%)$ y 17 pacientes tuvieron prescripciones de fármacos antiulcerosos de los 4 grupos farmacológicos simultáneamente (IBP, antiácidos, protectores de la mucosa y antagonistas H2) (Tabla 1). El 70\% de los pacientes polimedicados (42 461) tuvieron prescripción de al menos un fármaco gastrolesivo y el 17\% (10 140) prescripción simultánea de 2 o más fármacos gastrolesivos en el mismo mes. El grupo farmacológico gastrolesivo más prescrito en los pacientes polimedicados fueron los antiagregantes en el 52\% (27 078), seguido por los glucocorticoides y los AINE (Figura 2b).

En promedio, por mes, 280 pacientes tuvieron prescripción de 4 fármacos gastrolesivos simultáneamente; 28 pacientes, 5 o más gastrolesivos; y un paciente de 77 años fue prescrito con 7 fármacos gastrolesivos a la vez ( 3 glucocorticoides, 2 AINE, 2 ISRS). Del total de pacientes polimedicados gastroprotegidos, el 47\% (11 157) fueron ancianos, 35\% (8 308) adultos maduros, 13\% (3 086) adultos jóvenes y $5 \%\left(\begin{array}{l}1 \\ 187\end{array}\right)$ pacientes pediátricos. El 12\% (2 901) tuvieron asociado diagnósticos de riesgo gastrointestinal superior y 24\% (5 788) no tuvieron diagnósticos que justificaran el uso de antiulcerosos (Figura 3). El 48\% de los pacientes gastroprotegidos (11 182) no tuvieron gastrolesivos y en 13\% (3 370) estuvo justificado el uso de antiulcerosos al estar en tratamiento con 2 o más gastrolesivos. El 88\% de los pacientes con anticoagulantes (1 116),

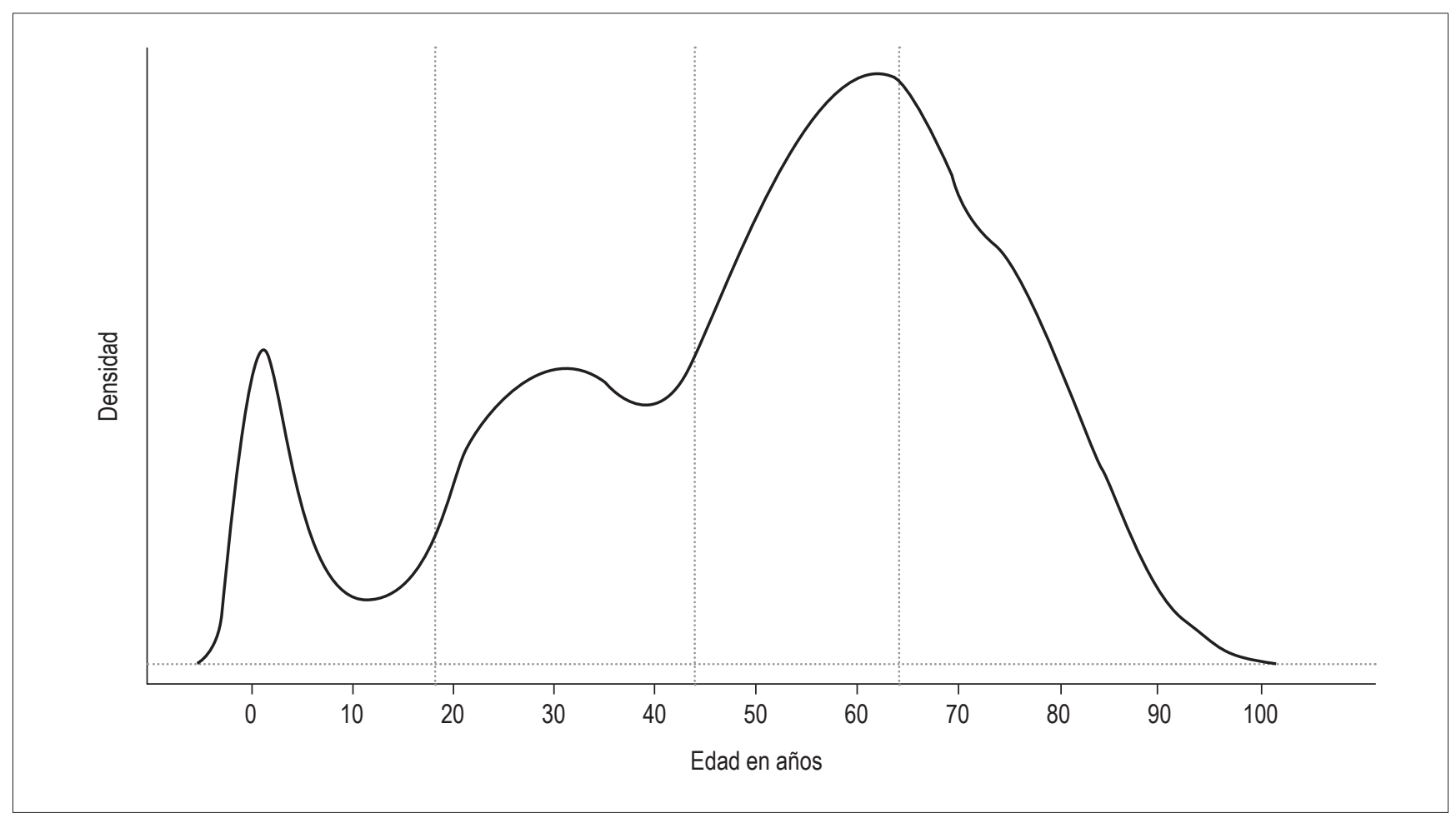

Figura 1. Densidad poblacional de pacientes polimedicados. 


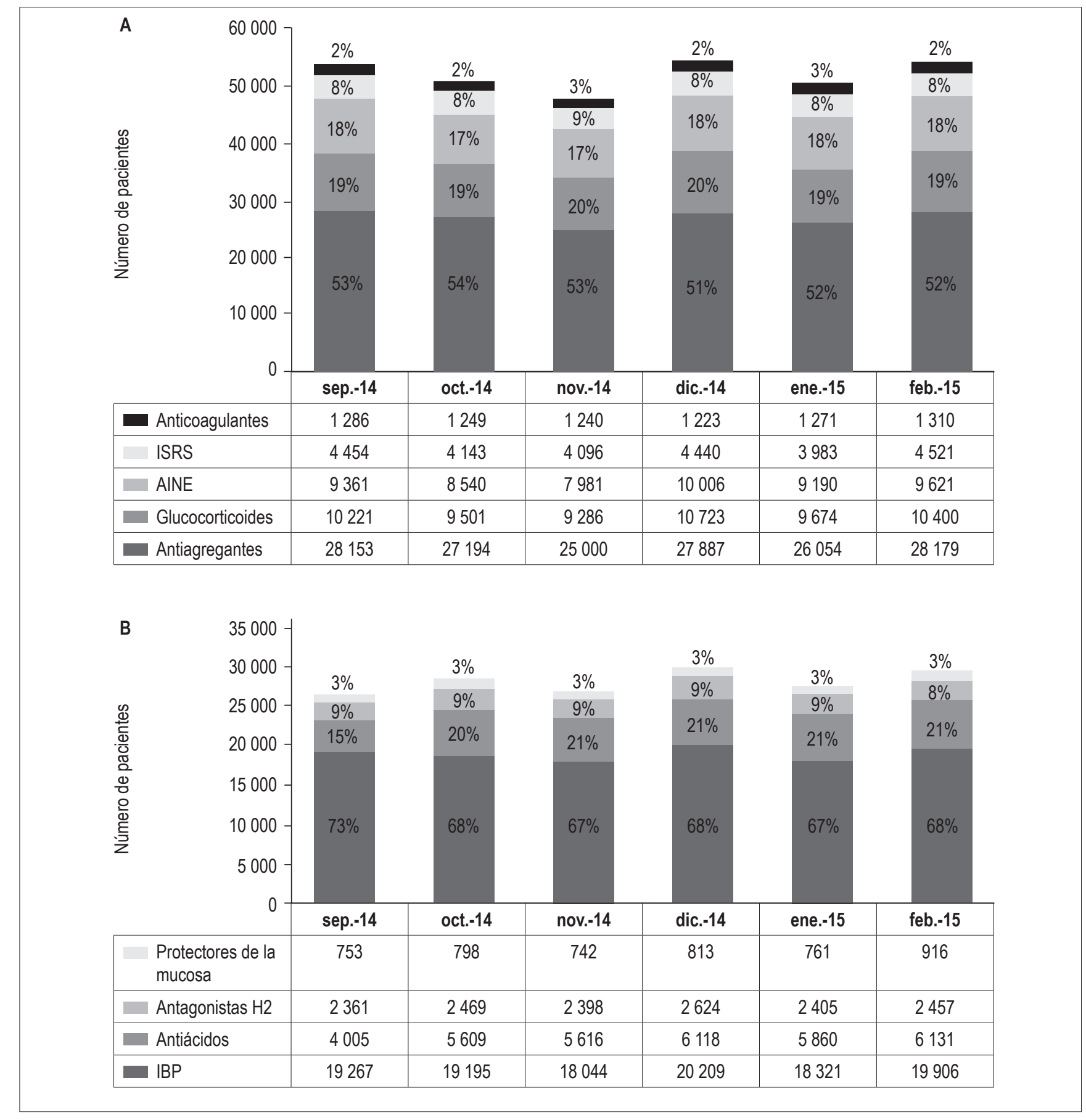

Figura 2. Porcentaje de uso por grupo farmacológico por mes en pacientes con polifarmacia. a) Fármacos antiulcerosos; b) Fármacos gastrolesivos. AINE: antinflamatorios no esteroideos; H2: receptor de histamina tipo 2; IBP: Inhibidores de la bomba de protones; ISRS: inhibidores selectivos de la recaptación de serotonina.

el $35 \%$ con antiagregantes plaquetarios ( 8420 ), el 17\% con glucocorticoides ( 1645 ), el $11 \%$ con ISRS (452) y el $10 \%$ con AINE (924) estuvieron gastroprotegidos.

De acuerdo a la información transaccional de los pacientes, y a los criterios preestablecidos para justificar la gastro- protección $(10,11)$, la gastroprotección no estuvo justificada en el 35\% de los pacientes (8 364), lo cual representa aproximadamente $\$ 75$ millones de pesos colombianos (COP) mensuales en fármacos antiulcerosos no justificados; con el uso adecuado y justificado de estos en pacientes 


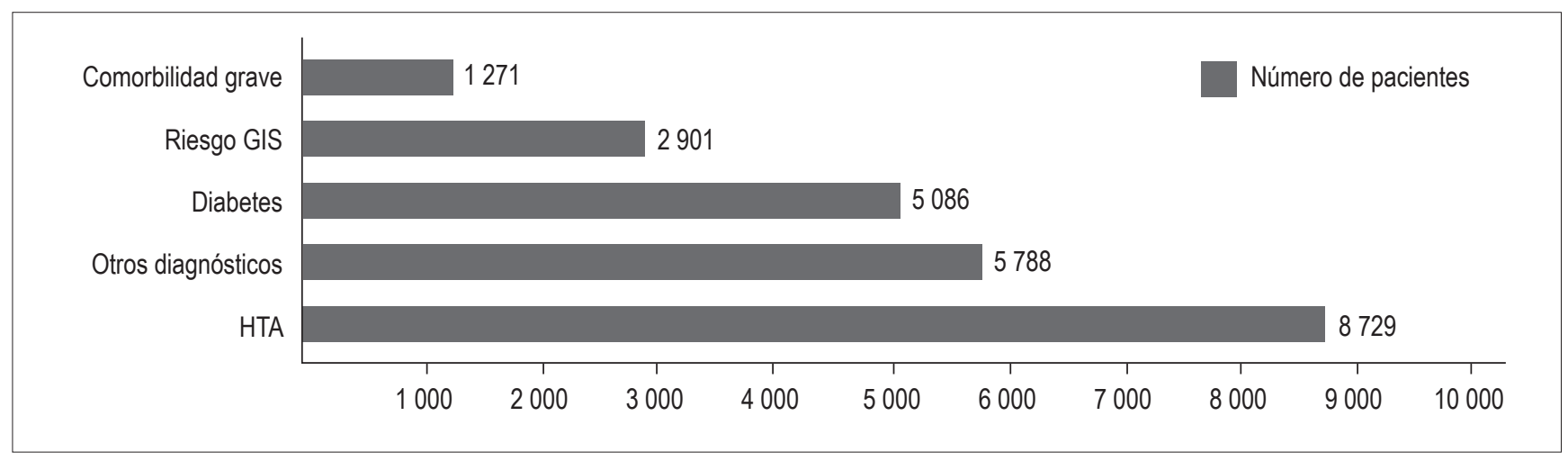

Figura 3. Diagnósticos asociados a la prescripción de antiulcerosos en pacientes con polifarmacia. Comorbilidad grave: septicemia, cáncer, hepatitis, insuficiencia renal crónica, traumatismos y quemaduras graves; Diabetes: diabetes mellitus tipo 1 y 2; HTA: hipertensión arterial; Otros diagnósticos: otros diagnósticos que no justifican el uso de antiulcerosos; Riesgo GIS: gastritis, duodenitis, esofagitis, ulceras gástrica, duodenal, péptica y gastroyeyunal, enfermedad de reflujo gastroesofágico, varices esofágicas, gástricas y duodenales y cánceres del tracto gastrointestinal superior.

Tabla 1. Pacientes con polifarmacia en tratamiento simultáneo con 2 o más antiulcerosos por mes y riesgo con el uso

\begin{tabular}{|c|c|c|c|c|c|c|c|c|}
\hline \multicolumn{9}{|c|}{ Pacientes con uso simultáneo de 2 grupos gastroprotectores } \\
\hline $\begin{array}{l}\text { Combinación de } \\
\text { gastroprotectores }\end{array}$ & Riesgo con el uso & sep-14 & oct-14 & nov-14 & dic-14 & ene-15 & feb-15 & $\begin{array}{l}\text { Promedio } \\
\text { ponderado }\end{array}$ \\
\hline IBP - antiácidos & Disminuye absorción IBP & 1891 & 2619 & 2643 & 2926 & 2820 & 2966 & 2682 \\
\hline IBP - protectores de la mucosa & Disminuye absorción IBP & 380 & 343 & 312 & 364 & 303 & 390 & 348 \\
\hline IBP - antagonistas H2 & - & 306 & 268 & 252 & 295 & 279 & 206 & 266 \\
\hline Antagonistas H2 - antiácidos & Disminuye absorción AntiH2 & 181 & 262 & 270 & 306 & 263 & 277 & 264 \\
\hline $\begin{array}{l}\text { Antagonistas } \mathrm{H} 2 \text { - protectores } \\
\text { de la mucosa }\end{array}$ & Disminuye absorción AntiH2 & 48 & 40 & 36 & 31 & 33 & 44 & 38 \\
\hline $\begin{array}{l}\text { Antiácidos - protectores de la } \\
\text { mucosa }\end{array}$ & Disminuye eficacia antiácido & 29 & 43 & 46 & 58 & 61 & 59 & 50 \\
\hline Total & 2.835 & 3575 & 3559 & 3980 & 3759 & 3942 & 3649 & \\
\hline \multicolumn{9}{|c|}{ Pacientes con uso simultáneo de 3 y 4 grupos gastroprotectores } \\
\hline $\begin{array}{l}\text { Combinación de } \\
\text { gastroprotectores }\end{array}$ & Riesgo con el uso & sep-14 & oct-14 & nov-14 & dic-14 & ene-15 & feb-15 & $\begin{array}{l}\text { Promedio } \\
\text { ponderado }\end{array}$ \\
\hline $\begin{array}{l}\text { IBP - antagonistas H2 - } \\
\text { antiácidos }\end{array}$ & $\begin{array}{l}\text { Disminuye absorción IBP y } \\
\text { antiH2 }\end{array}$ & 77 & 156 & 143 & 166 & 151 & 130 & 142 \\
\hline $\begin{array}{l}\text { IBP - antiácidos - protectores } \\
\text { de la mucosa }\end{array}$ & $\begin{array}{l}\text { Disminuye absorción IBP y } \\
\text { eficacia del antiácido }\end{array}$ & 73 & 135 & 143 & 140 & 128 & 156 & 134 \\
\hline $\begin{array}{l}\text { IBP - antagonistas H2 - } \\
\text { protectores de la mucosa }\end{array}$ & $\begin{array}{l}\text { Disminuye absorción IBP y } \\
\text { antiH2 }\end{array}$ & 15 & 18 & 8 & 11 & 17 & 20 & 15 \\
\hline $\begin{array}{l}\text { antagonistas H2 - antiácidos - } \\
\text { protectores de la mucosa }\end{array}$ & $\begin{array}{l}\text { Disminuye absorción antiH2 y } \\
\text { eficacia del antiácido }\end{array}$ & 7 & 10 & 7 & 8 & 6 & 9 & 8 \\
\hline $\begin{array}{l}\text { antagonistas } \mathrm{H} 2 \text { - antiácidos } \\
\text { - IBP - protectores de la } \\
\text { mucosa }\end{array}$ & $\begin{array}{l}\text { Disminuye absorción IBP y } \\
\text { antiH2 y eficacia del antiácido } \\
\text { (fallo terapéutico generalizado) }\end{array}$ & 10 & 15 & 14 & 11 & 14 & 17 & 14 \\
\hline Total & 182 & 334 & 315 & 336 & 316 & 332 & 313 & \\
\hline
\end{tabular}

H2: receptor de histamina tipo 2; IBP: Inhibidores de la bomba de protones. 
con polifarmacia se obtendría un ahorro estimado de $\$ 450$ millones de pesos colombianos en 6 meses. El cálculo de los coeficientes de correlación de Pearson y de Phi no encontró ninguna correlación entre la prescripción de fármacos antiulcerosos y alguno de los factores que justifican su prescripción (edad $\geq 65$ años, consumo de 2 o más gastrolesivos y/o diagnóstico de riesgo gastrointestinal), los coeficientes calculados estuvieron cercanos al 0 , con una distancia menor al 30\% de este. Los OR estimados confirmaron este resultado (Tabla 2), ningún factor por sí solo aumentó la probabilidad de prescripción de fármacos antiulcerosos en pacientes con polifarmacia, únicamente la presencia de 2 o más fármacos gastrolesivos en pacientes mayores o iguales a 65 años aumentó la probabilidad de prescripción de un fármaco antiulceroso en 1,2 veces (OR: 1,19; IC 95\%: $1,13-1,24)$ y la presencia de 2 o más fármacos gastrolesivos en pacientes mayores o iguales a 65 años con diagnóstico de riesgo gastrointestinal superior aumentó la probabilidad de prescripción de un fármaco antiulcerosos en 1,1 veces (OR: 1,13; IC 95\%: 1,01-1,27).

\section{DISCUSIÓN}

Guthrie B. y colaboradores, entre 1995-2010 en el Reino Unido, revelaron que los pacientes con 5 a 9 medicamentos aumentaron de $9,7 \%$ a $16,3 \%$, de 10 a 14 medicamentos de $1,5 \%$ a $4,7 \%$, y mayor o igual a 15 medicamentos, de $0,2 \%$ a $1,1 \% 18$ cifras que coinciden con los IP en este estudio ( $18,9 \%$ para $\geq 5$ fármacos; $2,1 \% \geq 10$ fármacos y $0,2 \%$ para $\geq 15$ fármacos). Qato D y colaboradores, al igual que en el presente estudio, encontraron que el $30 \%$ de los pacientes polimedicados eran $\geq 65$ años (19) y 1 de cada 5 fármacos usados en estos pacientes pueden ser inapropiados (20), adicional a esto, Eiras A. y colaboradores indican una pro- babilidad 4 veces mayor de tener medicamentos inapropiados si el paciente tiene polifarmacia (21), trayendo consigo mayor riesgo de RAM (22); la población de éste estudio tiene una alta probabilidad de tener fármacos inapropiados al ser polimedicados entre los 50 y 70 años.

Guthrie B. y colaboradores evaluaron la asociación entre el número de medicamentos y la probabilidad de presentar interacciones farmacológicas serias (IFS); 80,8\% pacientes con $\geq 15$ medicamentos tuvo potenciales IFS (18). Otros estudios encontraron asociación entre la polifarmacia y riesgo de hospitalizaciones (2,23-25), sin obtener mayor tasa de supervivencia, en comparación de los pacientes con menor número de medicamentos (25); por lo tanto, los 49 pacientes de nuestro estudio con $\geq 20$ medicamentos tuvieron alta probabilidad de IFS y hospitalización. El IP hallado por Eiras A (23,9\%) (23) fue cercano al encontrado en este estudio (22,3\%). Los antiulcerosos fueron prescritos en el $31,8 \%$ (23); a pesar del similar IP, nuestro estudio mostró mayor uso de antiulcerosos (40\%). Los IBP fueron los más empleados, de acuerdo a lo mencionado en estudios que recomiendan al omeprazol como primera línea en profilaxis de úlcera inducida por fármacos (10) al reducir el riesgo en un 50\% (11).

De resaltar la prescripción simultánea de $\geq 2$ antiulcerosos; no hay guías ni estudios que recomienden o justifiquen el uso profiláctico de varios medicamentos antiulcerosos al mismo tiempo; resulta debatible entonces, el tratamiento recibido por 300 pacientes polimedicados con prescripciones de $\geq 3$ medicamentos antiulcerosos, tratamiento que si bien no conlleva un beneficio adicional al paciente, si trae consigo un mayor riesgo de IFS, cuyo desenlace puede ser RAM o fallos terapéuticos de otros fármacos (al modificar la absorción de otros fármacos aumentando el $\mathrm{pH}$ gástrico) (26), que conlleven a hospitalizaciones. Las interaccio-

Tabla 2. Asociación entre la prescripción de antiulcerosos y los factores que justifican su prescripción (edad $\geq 65$ años, consumo de 2 o más gastrolesivos y la presencia de un diagnóstico de riesgo gastrointestinal superior)

\begin{tabular}{lcc}
\hline \multicolumn{1}{c}{ Factores que justifican la gastroprotección } & OR a & IC 95\% \\
\hline Edad $\geq 65$ años & 0,89 & $0,86-0,92$ \\
Diagnóstico de riesgo GIS & 0,98 & $0,90-1,07$ \\
Prescripción de $\geq 2$ gastrolesivos & 0,88 & $0,85-0,91$ \\
Edad $\geq 65$ años y diagnóstico de riesgo GIS & 0,34 & $0,31-0,38$ \\
Edad $\geq 65$ años y prescripción de $\geq 2$ gastrolesivos & 1,19 & $1,13-1,24$ \\
Diagnóstico de riesgo GIS y prescripción de $\geq 2$ gastrolesivos & 0,98 & $0,88-1,10$ \\
Edad $\geq 65$ años, diagnóstico de riesgo GIS y prescripción de $\geq 2$ gastrolesivos & $1,13 \quad++$ & $+1,01-1,27$ \\
Niveles de significancia (NS) & ++++ \\
\hline
\end{tabular}

a OR: odds Ratio

b NS: nivel de significancia, a menor nivel de significancia más veracidad del intervalo de confianza (IC)

GIS: gastrointestinal superior 
nes que se producen por el uso de IBP o antiH2 pueden persistir aun si se administran con tiempo de separación, debido al amplio tiempo de vida media de estos fármacos (ranitidina 6-8 h, omeprazol $>72 \mathrm{~h}$ ) (26). Además, el uso simultáneo de sucralfato con antiácidos que contienen aluminio debe usarse con precaución, debido a que este también contiene aluminio, lo cual incrementaría el potencial de toxicidad (27).

La gastroprotección reduce la morbimortalidad por uso de AINE y ASA a largo plazo (28). Ibáñez L y colaboradores (29) reportan $14,5 \%$ de casos de hemorragia de vías digestivas altas (HVDA) con antiagregantes plaquetarios, con incidencia de 50,6 casos/millón/año para ácido acetil salicílico (ASA) a dosis bajas. La incidencia reportada de HVDA por AINE: 152 casos/millón/año (30), notoriamente mayor; en nuestro estudio estaban gastroprotegidos el $31 \%$ de los pacientes con antiagregantes y solo un $10 \%$ de los pacientes con AINE, lo cual evidencia una posible infrautilización, concordante con otros estudios donde se ha observado infrautilización de gastroprotección en pacientes con AINE (11,31). Solo $12 \%$ de pacientes con fármacos gastrolesivos tenían justificado el uso de antiulcerosos, de acuerdo a la información transaccional existente y los criterios preestablecidos para justificar la gastroprotección $(10,11)$; esto puede llevar a suponer que pacientes que sí requieren gastroprotección no la reciben y pacientes que no la requieren la utilizan inadecuadamente.

El 24\% de los pacientes polimedicados gastroprotegidos no tenían asociado diagnóstico que justificara el uso de fármacos gastroprotectores en su información transaccional y solo el $12 \%$ tenían registro del diagnóstico de patología gastrointestinal superior de base; porcentaje muy por debajo de los reportados en estudios epidemiológicos, los cuales muestran una prevalencia de enfermedad ácido-péptica del $41 \%$ y de reflujo gastroesofágico en el $23 \%$ de los pacientes $(32,33)$. Este hallazgo puede estar asociado al registro inadecuado del diagnóstico por el médico prescriptor. Resulta evidente, entonces, que la calidad de los resultados del presente estudio depende de la calidad de los registros transaccionales. Por ende, puede estarse subestimando el número de pacientes con diagnósticos o antecedentes de patología gastrointestinal superior que justificasen el uso de gastroprotección debido a la ausencia del registro del mismo.

Santiestevea P. y colaboradores encontraron un porcentaje de gastroprotección inadecuada del $28,2 \%$ en pacientes con prescripción de AINE en Barcelona (34); resultados similares a los observados en el presente estudio donde se encontró un porcentaje de gastroprotección inadecuada del 35\%; este resultado representa $\$ 75$ millones de pesos colombianos mensuales. No se encontró ninguna asocia- ción importante entre la prescripción de gastroprotectores y alguno de los factores que justifican su prescripción, dejando abierta la posibilidad de que se estén prescribiendo antiulcerosos por la presencia de polifarmacia per se y no por los factores de riesgo a nivel gastrointestinal superior. Se recomienda entonces utilizar gastroprotección en pacientes con más de un gastrolesivo y con factores de riesgo gastrointestinal de base, independientemente de si son polimedicados o no.

Existen varias herramientas que apoyan la optimización de la medicación y la reducción de la prescripción; por ejemplo, los criterios STOPP y los criterios de Beers (35) en el marco del seguimiento farmacoterapéutico y las intervenciones farmacéuticas, las cuales han demostrado reducir el número medio de medicamentos prescritos en un 53\% (RR: 0,47) mejorando los resultados clínicos y la calidad de vida de los pacientes (36). La principal limitación del estudio es la calidad de la fuente de información; lo cual trae consigo una probabilidad de baja calidad en el registro del diagnóstico médico y ausencia de registro de antecedentes y patologías de base en pacientes crónicos, que conlleva a una subestimación de los pacientes que requerirían gastroprotección. Además, el desconocimiento de RAM o fallos terapéuticos presentados en la población de estudio, así como los desenlaces clínicos de los pacientes cuya gastroprotección no fue adecuada, bien sea por exceso o por defecto, son otras limitaciones del presente estudio. Sin embargo, los hallazgos aquí encontrados abren las puertas a futuras investigaciones que demuestren la asociación existente entre la polifarmacia y la gastroprotección, y exhiban las consecuencias de una gastroprotección inadecuada en la calidad de vida de los pacientes, los costos asistenciales, el impacto financiero en el sistema de salud, entre otros.

\section{Agradecimientos}

Agradecemos a MC21 Colombia por suministrar la información con la cual se realizó el presente estudio y a Camilo Yate, estadístico del departamento clínico, por el análisis aplicado a los datos, sus aportes y comentarios para la realización de este artículo.

\section{Fuente de apoyo financiero}

Tanto la financiación como las bases de datos transaccionales, de las cuales se obtuvo la información de esta investigación, fueron brindadas por MC21 Colombia SAS. Sin embargo, los autores han declarado que no existe conflicto de intereses personales o de financiamiento en la realización del presente trabajo. 


\section{REFERENCIAS}

1. Hung WW, Ross JS, Boockvar KS, Siu AL. Recent trends in chronic disease, impairment and disability among older adults in the United States. BMC Geriatr. 2011;11:47.

2. Jokanovic N, Tan ECK, Dooley MJ, Kirkpatrick CM, Bell JS. Prevalence and factors associated with polypharmacy in long-term care facilities: a systematic review. J Am Med Dir Assoc. 2015;16(6):535.e1-12.

3. Bourgeois FT, Shannon MW, Valim C, Mandl KD. Adverse drug events in the outpatient setting: an 11-year national analysis. Pharmacoepidemiol Drug Saf. 2010;19(9):901-10.

4. Hovstadius B, Petersson G. The impact of increasing polypharmacy on prescribed drug expenditure-a register-based study in Sweden 2005-2009. Health Policy. 2013;109(2):166-74.

5. Fulton MM, Allen ER. Polypharmacy in the elderly: a literature review. J Am Acad Nurse Pract. 2005;17(4):123-32.

6. Ramírez-Duque N, Rivas-Cobas C, Bernabeu-Wittel M, Ruiz-Cantero A, Murcia-Zaragoza J, Oliver M, et al. [Drug prescription profile in patients with advanced chronic diseases]. Rev Esp Geriatr Gerontol. 2014;49(6):255-9.

7. Cho HJ, Kim CB. Prescription behaviours of office-based doctors to standardized common cold patients in Korea. Pharmacoepidemiol Drug Saf. 2002;11(5):401-5.

8. Kim S. An analysis of effects by delisting non-ethical gastrointestinal drugs. In College of Public Health. Seoul National University: Seoul, 2008.

9. Cho E, Sukyeong K. Prescribing Superfluous Gastroprotective Agents: an Indicator of Polypharmacy. Kor J Clin Pharm. 2011;21(2):156-60.

10. Vallès Fernández R, Franzi Sisó A, Ferro Rivera J. Condiciones clínicas y terapéuticas que requiere gastroprotección. FMC. 2014;21(9):528-33.

11. Grupo de Trabajo del Sector Zaragoza I SALUD. Guía de Práctica Clínica de empleo de los inhibidores de la bomba de protones en la prevención de gastropatías secundarias a fármacos. Unidad docente de medicina familiar y comunitaria. Zaragoza. 2012.

12. Información Farmacoterapéutica de la Comarca. Inhibidores de la bomba de protones: ¿se puede vivir sin ellos? 2010; 18(3).

13. Martin $A B$, Lassman $D$, Washington $B$, Catlin A, Team NHEA, others. Growth in US health spending remained slow in 2010; health share of gross domestic product was unchanged from 2009. Health Affairs. 2012;31(1):208-19.

14. $\mathrm{R}$ Core Team. $\mathrm{R}$ : A language and environment for statistical computing. R. 2015. http://www.R-project.org/.

15. Dates and Times Made Easy with lubridate | Grolemund | Journal of Statistical Software [Internet]. [citado 20 de mayo de 2016]. Recuperado a partir de: https://www.jstatsoft. org/article/view/v040i03

16. Grothendieck G. sqldf: Perform SQL Selects on R Data Frames [Internet]. 2014 [citado 20 de mayo de 2016]. Recuperado a partir de: https://cran.r-project.org/web/ packages/sqldf/index.html
17. Robin X, Turck N, Hainard A, Tiberti N, Lisacek F, Sanchez J-C, et al. pROC: an open-source package for $\mathrm{R}$ and $\mathrm{S}+$ to analyze and compare ROC curves. BMC Bioinformatics. 2011;12:77.

18. Guthrie B, Makubate B, Hernandez-Santiago V, Dreischulte $\mathrm{T}$. The rising tide of polypharmacy and drug-drug interactions: population database analysis 1995-2010. BMC Med. 2015; $13: 74$

19. Qato DM, Alexander GC, Conti RM, Johnson M, Schumm P, Lindau ST. Use of prescription and over-the-counter medications and dietary supplements among older adults in the United States. JAMA. 2008;300(24):2867-78.

20. Roughead EE, Anderson B, Gilbert AL. Potentially inappropriate prescribing among Australian veterans and war widows/widowers. Intern Med J. 2007;37(6):402-5.

21. Eiras A, Teixeira MA, González-Montalvo JI, Castell M-V, Queipo R, Otero Á. Consumo de medicamentos en mayores de 65 años en Oporto (Portugal) y riesgo de prescripción de medicamentos potencialmente inapropiados. Atención Primaria. 2016;48(2):110-20.

22. Atkin PA, Veitch PC, Veitch EM, Ogle SJ. The epidemiology of serious adverse drug reactions among the elderly. Drugs Aging. 1999;14(2):141-52.

23. Cherubini A, Eusebi P, Dell'Aquila G, Landi F, Gasperini B, Bacuccoli R, et al. Predictors of hospitalization in Italian nursing home residents: the U.L.I.S.S.E. project. J Am Med Dir Assoc. 2012;13(1):84.e5-10.

24. Nguyen JK, Fouts MM, Kotabe SE, Lo E. Polypharmacy as a risk factor for adverse drug reactions in geriatric nursing home residents. Am J Geriatr Pharmacother. 2006;4(1):36-41.

25. Díez-Manglano J, Giménez-López M, Garcés-Horna V, Sevil-Puras M, Castellar-Otín E, González-García P, et al. Excessive polypharmacy and survival in polypathological patients. Eur J Clin Pharmacol. 2015;71(6):733-9.

26. De las Salas-Martínez RB,Villarreal-Cantillo E. Interacciones en el uso de antiácidos, protectores de la mucosa y antisecretores gástricos. Salud Uninorte. Barranquilla (Col.) 2013; 29 (3): 441-57.

27. Ritschel WA, Banerjee PS, Koch HP, Czeijka M. Cimetidine-sucralfate drug interaction. Methods Find Exp Clin Pharmacol. 1984;6(5):261-3.

28. Rostom A, Dube C, Wells G, Tugwell P, Welch V, Jolicoeur E, et al. Prevention of NSAID-induced gastroduodenal ulcers. Cochrane Database Syst Rev. 2002; (4):CD002296.

29. Ibáñez L, Vidal X, Vendrell L, Moretti U, Laporte JR, Spanish-Italian Collaborative Group for the Epidemiology of Gastrointestinal Bleeding. Upper gastrointestinal bleeding associated with antiplatelet drugs. Aliment Pharmacol Ther. 2006;23(2):235-42.

30. Laporte J-R, Ibáñez L, Vidal X, Vendrell L, Leone R. Upper gastrointestinal bleeding associated with the use of NSAIDs: newer versus older agents. Drug Saf. 2004;27(6):411-20.

31. Rostom A, Wells G, Tugwell P, Welch V, Dube C, McGowan J. Prevention of NSAID-induced gastroduodenal ulcers. Cochrane Database Syst Rev. 2000;(4):CD002296. 
32. Morini S, Zullo A, Oliveti D, Chiriatti A, Marmo R, Chiuri $\mathrm{DAE}$, et al. A very high rate of inappropriate use of gastroprotection for nonsteroidal anti-inflammatory drug therapy in primary care: a cross-sectional study.J Clin Gastroenterol. 2011;45(9):780-4.

33. Verdejo-Bravo C, Calleja-Panero JL, Guillén-Llera F, RiberaCasado JM. Estudio epidemiológico sobre la enfermedad ácido-péptica en pacientes ancianos ambulatorios. Rev Esp Geriatr Gerontol. 2006;41(1):21-8.

34. Del Piano M, Bianco MA, Cipolletta L, Zambelli A, Chilovi F, Di Matteo G, et al. The «Prometeo» study: online collec- tion of clinical data and outcome of Italian patients with acute nonvariceal upper gastrointestinal bleeding. J Clin Gastroenterol. 2013;47(4):e33-37.

35. Carrillo Santiesteve P, Amado Guirado E, de la Fuente Cadenas JA, Pujol Ribera E, Tajada C, Calvet S, et al. [Prescribing non-steroidal antiinflammatory drugs and gastrointestinal protection in primary care]. Aten Primaria. 2008;40(11):559-64.

36. Wilcock M, Hughes P. GP perceptions of medicines optimisation principles. Prescriber. 2014;25(18):13-7. 